https://helda.helsinki.fi

Changing trends in pediatric tonsil surgery

Sakki, Anniina

2019-03

Sakki , A , Mäkinen , L K , Roine , R P \& Nokso-Koivisto , J 2019 , ' Changing trends in pediatric tonsil surgery ' , International Journal of Pediatric Otorhinolaryngology , vol. 118 , pp. 84-89 . https://doi.org/10.1016/j.ijporl.2018.12.028

http://hdl.handle.net/10138/300802

https://doi.org/10.1016/j.ijporl.2018.12.028

publishedVersion

Downloaded from Helda, University of Helsinki institutional repository.

This is an electronic reprint of the original article.

This reprint may differ from the original in pagination and typographic detail.

Please cite the original version. 


\title{
Changing trends in pediatric tonsil surgery
}

\author{
Anniina Sakki ${ }^{\mathrm{a}, *}$, Laura K. Mäkinen ${ }^{\mathrm{a}}$, Risto P. Roine ${ }^{\mathrm{b}, \mathrm{c}}$, Johanna Nokso-Koivisto ${ }^{\mathrm{a}}$ \\ ${ }^{a}$ Department of Otorhinolaryngology - Head and Neck Surgery, Helsinki University Hospital, P.O. Box 263 (Kasarmikatu 11-13), FI-00029, HUS, Finland \\ ${ }^{\mathrm{b}}$ Helsinki and Uusimaa Hospital Group, Group Administration, P.O. Box 705, FI-00029, HUS, Finland \\ ${ }^{\mathrm{c}}$ University of Eastern Finland, Department of Health and Social Management, P.O. Box 1627, FI-70211, Kuopio, Finland
}

\section{A R T I C L E I N F O}

\section{Keywords:}

Tonsil surgery

Tonsillotomy

Tonsillectomy

Children, Healthcare costs

\begin{abstract}
A B S T R A C T
Objectives: We analyzed trends in tonsil surgery over a 10-year period in a single tertiary care hospital and evaluated the effects of these changes on use of hospital services and healthcare costs.

Methods: This was a retrospective cohort study based on data from databases at the Department of Otorhinolaryngology, Helsinki University Hospital, Helsinki, Finland. Children under 16 years of age with tonsillectomy (TE) or tonsillotomy (TT) performed during 2007-2016 were included in the study.

Results: In 10 years, 4979 tonsil surgeries were performed on 4951 children: TE in 3170 (64\%) and TT in 1781 (36\%) children. The total number of tonsil surgeries stayed nearly constant. TT operations commenced in the study hospital in 2009 and from 2012 onwards have been more common than TE procedures. Altogether 279 patients visited the emergency department because of complications; TE patients had 9.0 visits/100 surgeries and TT patients 1.8 visits/100 surgeries. The most common complication was postoperative hemorrhage: 200 cases $(6.3 \%)$ in the TE group and 11 cases $(0.6 \%)$ in the TT group. During the two-year follow-up after tonsil surgery the total costs of healthcare services were significantly lower in the TT group than in the TE group. Conclusion: Considerable changes have occurred in tonsil surgery in children during the 10-year study period; TT is today performed more often than TE. As a consequence, complications, readmissions to hospital, and number of patients treated in the operating room because of postoperative hemorrhage have decreased, lowering the costs of healthcare.
\end{abstract}

\section{Introduction}

Tonsil surgery has a long history; removal of tonsillar tissue was first reported over 3000 years ago [1]. Over the past centuries, tonsil surgery has undergone a number of changes, in both indications and surgical methods. Before 1900, the main surgical technique was tonsillotomy (TT), the enucleation of the tonsil with the finger, followed by the presentation of the "tonsil guillotine", which was used to cut off the tonsil. As anesthesia improved, dissection tonsillectomy (TE) became the method of choice, with recurrent tonsillitis being the most prevalent indication $[1,2]$. However, development of antibiotics and an increased awareness of tonsil-related sleep-disordered breathing (SDB) in children have made upper airway obstruction the most common indication for tonsil surgery in the last 30 years [2]. In 1999, Linder et al. [3] and Hultcrantz et al. [4] reintroduced the previously abandoned method of TT; compared with TE, TT was associated with less morbidity such as postoperative pain and hemorrhage. The positive results have been confirmed later [5-7], and a shift from TE to TT in treating SDB in children has taken place in various countries [8].
Even though many studies have shown the advantages of TT compared with TE, there are still some concerns related to this technique. The remaining tonsillar tissue might regrow and eventually lead to the need for resurgery. It has also been proposed that the physiology of the remaining tonsil might be altered, leading to chronic tonsillitis and peritonsillar abscesses [9,10].

As the method for one of the most common surgical procedures performed in otorhinolaryngology, tonsil surgery, has changed, it is essential to evaluate the impact of the change on patients and on healthcare costs. The aim of this study was to analyze the changes in tonsil surgery in children at a single tertiary care center over a 10-year period and the effects of these changes on the number of complications, emergency department visits, readmissions to hospital after tonsil surgery, and costs of healthcare. Furthermore, the number of reoperations and incidence of peritonsillar abscesses after TT were explored.

\section{Methods}

This is a retrospective cohort study based on data from the

\footnotetext{
* Corresponding author.

E-mail address: anniina.sakki@helsinki.fi (A. Sakki).
} 




Fig. 1. Number of tonsil surgeries performed on children aged less than 16 years at the Department of Otorhinolaryngology, Helsinki University Hospital, in 2007-2016.

databases of the Department of Otorhinolaryngology - Head and Neck Surgery (ORL) of the Helsinki University Hospital (HUH), Helsinki, Finland. Children younger than 16 years were included in the study. Routinely, all tonsil surgeries in children are done as day-surgeries unless significant comorbidity requires postoperative follow-up at the ward.

Two different searches from two separate databases were done. The first data, retrieved from the surgical database, included all children less than 16 years of age operated on with TE or TT (with or without adenotomy) at the Department of ORL from January 2007 to December 2016. The search was based on surgical procedure codes (Nordic Medico-Statistical Committee Classification of Surgical Procedures) covering TE and TT with or without adenotomy (EMB10, EMB20, EMB15). The indications and number of tonsil surgeries and resurgeries were recorded.

The second search was from the patient database covering all children aged less than 16 years who visited the emergency polyclinic at the Department of ORL because of tonsil surgery complications from January 2007 to December 2016. The search was based on ICD diagnosis codes (International Statistical Classification of Diseases and Related Health Problems, ICD) covering complications of tonsil surgery: hemorrhage and hematoma complicating a procedure (T81.0), infection following a procedure (T81.4), other post-procedural states (Z98.8), and peritonsillar abscess (J36). Demographic and clinical data (age, sex, surgical technique, previous tonsillar procedures, inpatient days because of complication, and postoperative hemorrhages with returnto-theatre) were retrieved from patient charts. Costs of complications were estimated from performance-based fees of the hospital.

Hospital costs covered the time from the operation until the end of a two-year follow-up and were obtained from the Ecomed clinical patient administration system (Datawell Ltd., Espoo, Finland), where data on hospital treatment costs of individual patients are stored. The search was done with ID numbers of patients operated on at the Department of ORL, HUH in 2007-2016 and includes costs of the operative period and public healthcare ORL specialty visits in the HUH region during the follow-up. The costs comprise the polyclinic visits (emergency and referral), inpatient days, primary surgeries, resurgeries, and other possible ORL surgeries, laboratory tests, imaging, and histopathological analyses.

\subsection{Tonsillotomy}

The technique for TT was adopted from Hultcrantz et all [7]. The used radiofrequency generator was BM-780 II (Sutter Medizintechnik $\mathrm{GmbH}$, Freiburg, Germany). A small amount of lidocaine-adrenaline was injected in the tonsillar tissue. With a bent needle electrode (ARROWtip, Re-Usable, Sutter Medizintechnik GmbH, Freiburg, Germany) and $80 \mathrm{~W}$ effect the protruding part of the tonsil was cut off in the plane of the pillars. The hemostasis was achieved with bipolar diathermy.

\subsection{Tonsillectomy}

TE was performed using cold instruments and hemostasis achieved with the bipolar diathermy.

\subsection{Ethics}

The study protocol was approved, and institutional research permission was granted by the Department of ORL, HUH, Helsinki, Finland.

\subsection{Statistics}

Statistical analyses were performed using SPSS software (version 22, SPSS Inc., Chicago, IL, USA). Categorical variables are reported as frequencies and percentages, and continuous variables as means (standard deviation, SD) or medians. Pearson Chi-squared test or independent samples $t$-test was used to compare the groups. $P$ values < 0.05 were considered significant.

\section{Results}

In 10 years, altogether 4979 tonsil surgeries were performed on 4951 children younger than 16 years at the Department of ORL, HUH. Among these children, the initial tonsil surgery method was TE in 3170 (64\%) and TT in 1781 (36\%). Of TE and TT children, 52\% and 56\%, respectively, were males. The median age at surgery was higher in the TE group (7.6 years, SD 2.6, range 0.6-16.0) than in the TT group (5.3 years, SD 4.0, range 1.2-15.8).

The increase in TTs began in 2009, and from 2009 to 2013 the proportion of TT of all tonsil surgeries increased from $7.7 \%$ to $61.6 \%$ (Fig. 1). From 2012 onwards, TT was more common than TE. A slight 
annual decrease in the total number of tonsil surgeries can be seen after the year 2011 .

There were no changes in indications of tonsil surgery in children during the study period. The most frequent indication for tonsil surgery was obstructive symptoms because of tonsillar hypertrophy (74\% of all tonsil surgeries), followed by infection (24\%).

The biggest change in the surgery method was seen in the treatment of SDB; after 2011, TT was performed more often than TE, and $61 \%$ of all procedures were TTs in 2016.

\subsection{Resurgery}

A total of 28 patients ( $0.6 \%$ of all children with tonsil surgery) underwent a reoperation of the tonsils during the study period; two patients had TE as the initial surgery $(0.06 \%$ of all TEs) and 26 patients had TT (1.5\% of all TTs). The indication of the initial surgery was SDB in both groups. The reoperation was TE in 26 patients and TT in two patients. The median time from the first surgery to the reoperation was 3.9 years in the TE group and 2.0 years in the TT group. Among the children who needed resurgery, the age at first surgery was less than four years in $73 \%$ in the TT group. The main reason for reoperation was the recurrence of upper airway obstruction because of tonsillar regrowth ( $68 \%$ of all reoperations). Regrowth of tonsillar tissue in combination with recurrent or chronic tonsillitis was the indication for $14 \%$ of reoperations. Only two patients needed resurgery because of peritonsillar abscess, both were in the TT group ( $0.1 \%$ of all TTs). The estimated cost of one tonsil surgery in 2018 is $1329 €$, hence, the total cost of reoperations after TT is approximately $34600 €$.

\subsection{Complications of tonsil surgery}

At the emergency polyclinic of the Department of ORL, the total number of visits due to a complication of tonsil surgery was 713 in children aged less than 16 years during the 10 -year study period. Of the patients, $364(51 \%)$ had been operated on in the private sector or in another public hospital of the HUH area and were excluded from this study. In addition, the visits after adenoidectomies without tonsil procedures $(n=30)$ were excluded. Thus, the total number of emergency department visits after TE or TT performed in our hospital was 319, and the total number of patients was 279 , as 30 patients (11\%) needed more than one visit at the emergency department. The median age of the children was 6.8 years (range 1.63-15.93), and 54\% were males. The indication of the initial surgery was SDB in all patients who visited at the emergency department.

After TE, there were 9.0 visits/100 surgeries and after TT 1.8 visits/ 100 surgeries at the emergency department. The most common complication was postoperative hemorrhage, accounting for 200 visits (6.3\% of all TEs) in the TE group and 11 visits $(0.6 \%$ of all TTs) in the TT group $(P<0.05)$ (Fig. 2). All but two of the bleedings were secondary (occurred $>24 \mathrm{~h}$ postoperatively). Other reasons for the visits were pain, infection, nausea, dehydration, fever, and peritonsillar abscesses. The number of patients needing return-to-theatre to control the bleeding was 69 , of these 65 ( $2 \%$ of all TEs) were in the TE group and 4 $(0.2 \%$ of all TTs) in the TT group.

Altogether 121 patients had a complication that necessitated hospital admission: 112 (3.5\% of all TEs) in the TE group and $9(0.5 \%)$ in the TT group (Table 1). There were 6.8 inpatient days/100 surgeries after TE and 0.7 inpatient days/100 surgeries after TT. No mortalities occurred during the study period. The total number of emergency department visits, days in hospital because of major complications, and postoperative hemorrhages requiring return-to-theatre (RTT) are presented in Fig. 3. In our hospital, the total number of tonsil surgeries (TE and TT) stayed approximately the same, but the number of complications, emergency department visits, and inpatient days because of complications due to tonsil surgery decreased during the study period.

Costs of complications were estimated by using performance-based fees of the hospital. Most surgeries were performed without any complications. The costs caused by complications were divided by the number of surgeries to estimate the average costs of complications (Fig. 4). The average costs of complications were $128 €$ less per surgery after TT than after TE, hence, the approximate total savings resulting from the change of the operative method were $230000 €$ with the 1787 TTs performed from 2009 to 2016.

During the two-year follow-up after tonsil surgery the total costs of healthcare services (including those of the operative period) were significantly smaller in the TT group than in the TE group (Fig. 5). The costs of polyclinic visits were higher in the TT group than in the TE group, but otherwise the costs were lower in the TT group. The average total costs in the TT group were $132 €$ less per surgery than in the TE group.

\section{Discussion}

This study describes the changes in trends of tonsil surgery at a single tertiary care center and the effects of these changes. Currently, TT is performed more often than TE in the treatment of airway obstruction caused by tonsillar hypertrophy in children younger than 16 years at the study hospital. The change of the surgical method from TE to TT has decreased the complication rates of tonsil surgery, reducing the number of emergency department visits, costly inpatient days because of complications, and the number of postoperative hemorrhages requiring return-to-theatre.

TT operations were started at our department in 2009, and from 2012 onwards TT has been more common than TE in the treatment of airway obstruction in children. This change was initiated by studies establishing the safety and efficiency of TT [11-13]. In addition, TT has been reported to decrease the risk of postoperative morbidity, especially pain and hemorrhage $[7,14,15]$. Accordingly, in our study there were considerably fewer emergency department visits after TT than after TE (1.8 vs. 9.0 visits/100 surgeries). The most frequent indications for visits were postoperative hemorrhage, pain, and infection. A significant change was seen in the admissions to hospital because of a complication and the number of postoperative hemorrhages requiring return-to-theatre, which decreased markedly during the study period. Fewer complications result in a smaller workload for the hospital, decreased healthcare costs, and, most importantly, less individual morbidity. As postoperative hemorrhage associated with tonsil surgery in children is always a potentially lethal complication, any means to reduce this risk are beneficial.

Costs of complications were estimated from performance-based fees of the hospital and the average costs of complications were $128 €$ less per surgery after TT than after TE. Furthermore, during the two-year follow-up after tonsil surgery, total costs of healthcare services were significantly smaller in the TT group than in the TE group. In addition to the benefits of TT to the patient (less postoperative pain, less pain medication, faster recovery) $[16,17]$, TT seems to be beneficial also in reducing healthcare costs.

During the period from 2007 to 2016 approximately 500 tonsil surgeries were performed every year for children at our department. The incidence of tonsil surgeries remained relatively stable, in contrast to Sweden, where the rate of tonsil surgery has doubled over 26 years in children and adolescents [18]. A potential explanation for this difference is that tonsil surgeries in the Helsinki area are performed partly by the private sector, and hence, the exact number of tonsil surgeries is not known.

The most common indication for tonsil surgery in our study was obstructive symptoms, accounting for $73 \%$ of all tonsil surgeries. The incidence of infectious indications remained relatively stable. Similar findings have been published in other studies [18-20], reflecting an increased awareness of upper airway obstruction. TT is mostly performed because of obstructive symptoms, although a few studies have shown that TT can be beneficial also for children and young adults with 


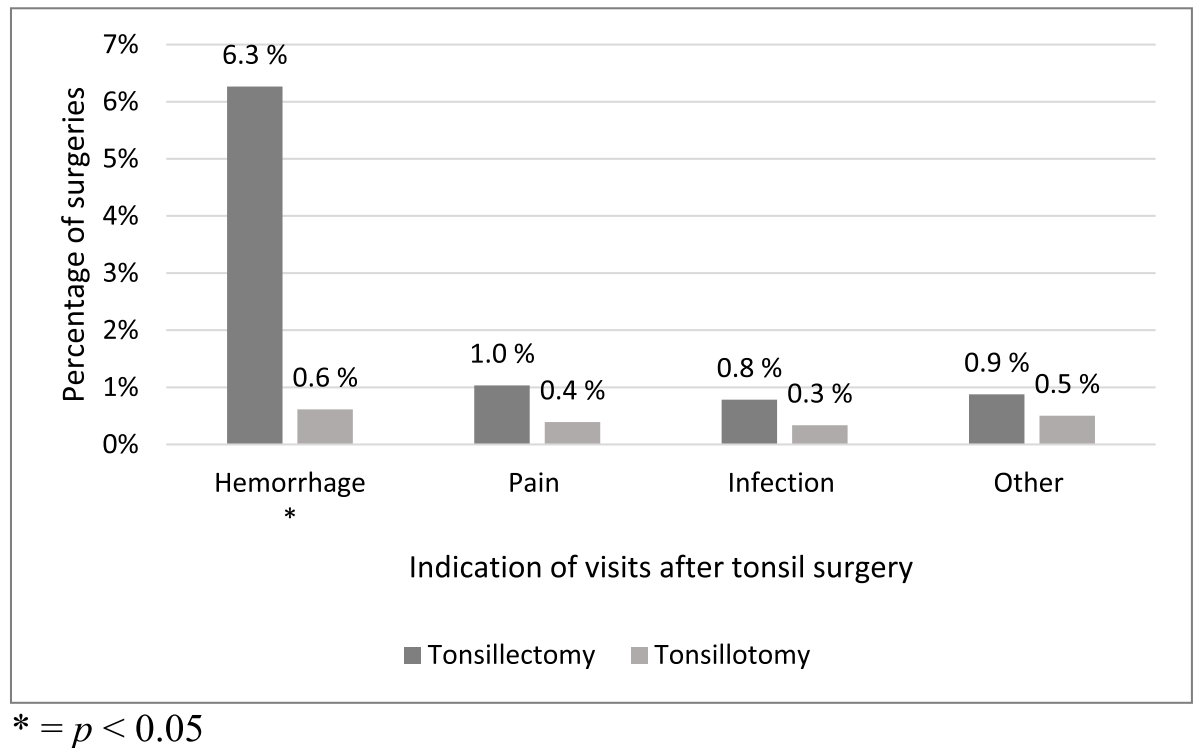


children under 16 years of age.

Table 1

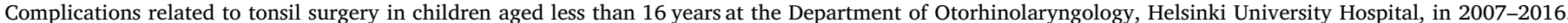

\begin{tabular}{|c|c|c|c|c|c|c|}
\hline & $\mathrm{TE}$ & $\%$ & TT & $\%$ & Total & $\%$ \\
\hline & $(\mathrm{N}=3192)$ & & $(\mathrm{N}=1787)$ & & $(\mathrm{N}=4979)$ & \\
\hline Postoperative visits at ER, total & 286 & $\mathrm{x}$ & 33 & $\mathrm{x}$ & 319 & $\mathrm{x}$ \\
\hline Postoperative visits at ER/100 surgeries & 9 & & 1.8 & & 6.4 & \\
\hline Visits due to postoperative hemorrhage & 200 & 6.3 & 11 & 0.6 & 211 & 4.2 \\
\hline Children readmitted to hospital & 112 & 3.5 & 9 & 0.5 & 121 & 2.4 \\
\hline
\end{tabular}

$\mathrm{TE}=$ tonsillectomy, $\mathrm{TT}=$ tonsillotomy, $\mathrm{ER}=$ emergency clinic of the Department of Otorhinolaryngology.

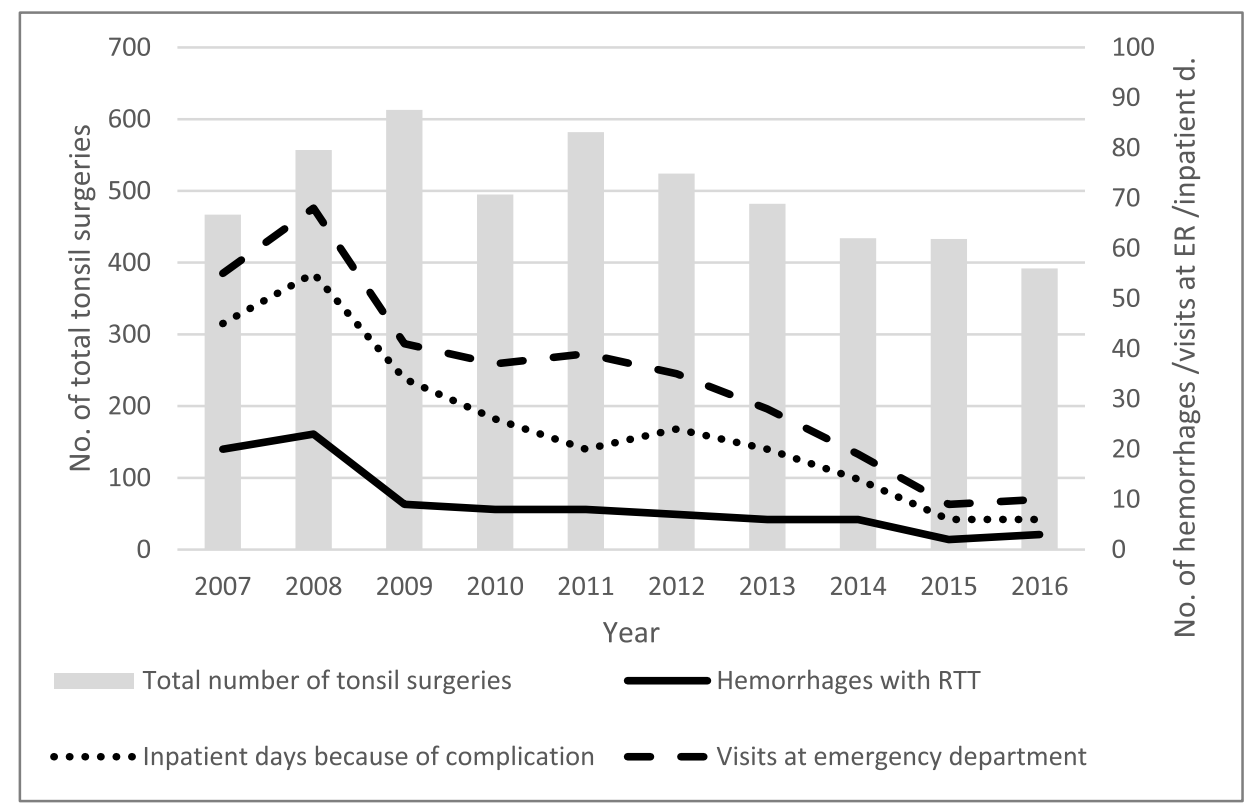

RTT=return to theatre, ER= emergency clinic of the Department of Otorhinolaryngology, $d .=$ day

Fig. 3. Changes in tonsil surgery complications in children over a 10-year period at the Department of Otorhinolaryngology, Helsinki University Hospital.

recurrent tonsillitis [21,22].

A known disadvantage of TT is regrowth of tonsils with recurrence of obstructive symptoms. In our study, resurgery was needed after TT in $1.5 \%$ of all TTs performed in eight years. This is in concordance with a recent review by Windfuhr et al. [23] covering 86 studies; secondary surgery was performed in $1.6 \%$ of the original 5877 TT cases. It seems that patients younger than three years have a bigger risk of regrowth of the tonsillar tissue $[9,24]$. In our study, the age at the time of first 


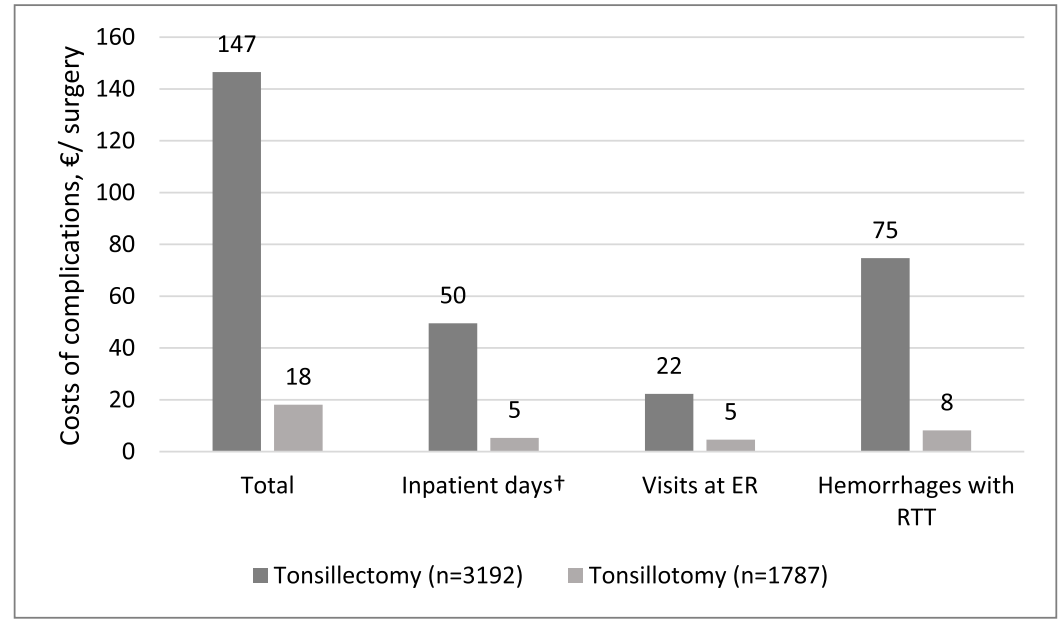

Inpatient days because of complication, ER=emergency clinic of the Department of Otorhinolaryngology, RTT=return to theatre

Fig. 4. Average costs of complications per tonsil surgery at the Department of Otorhinolaryngology, Helsinki University Hospital, in 2007-2016.

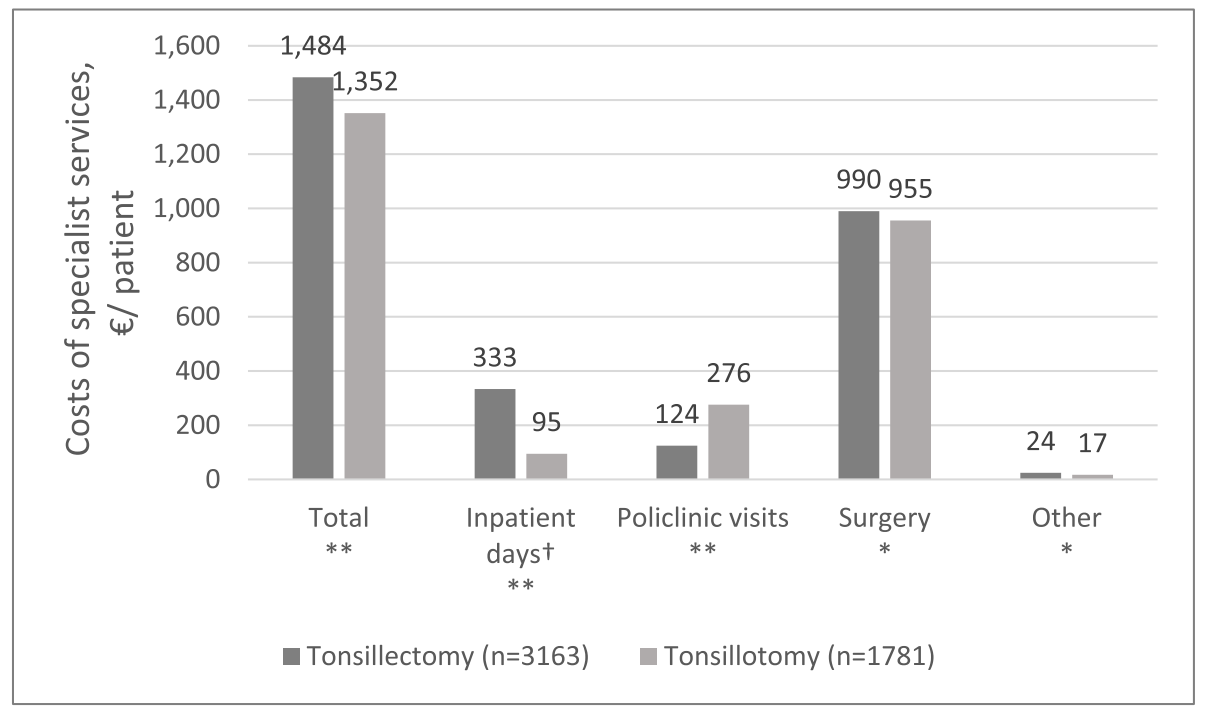

$\dagger$ Inpatient days because of complication, $* *=p<0.001, *=p<0.05$

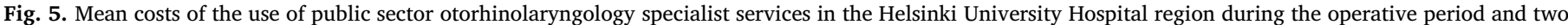
years postoperatively in children operated on at the Department of Otorhinolaryngology, Helsinki University Hospital, in 2007-2016.

surgery was less than three years in $50 \%$ of children who needed resurgery in the TT group. It is important that benefits and possible harms and risks of the operation are discussed with the patient and parents before surgery.

Historically, there have been concerns that after TT the physiology of the remaining tonsil might be altered, leading to tonsilloliths, infections, and peritonsillar abscesses. In our study, upper airway obstruction was the most common reason for reoperation. Regrowth of tonsillar tissue with chronic and/or recurrent tonsillitis was a less frequent indication for secondary TE, and there were only two patients who underwent revision surgery due to postoperative peritonsillar abscess. Ericsson et al. [6] have also reported no increase in the incidence of throat infections three years after TT. As chronic and/or recurrent tonsillitis and disturbing tonsilloliths are more common among adolescents and young adults, follow-up studies covering these age groups are needed to determine the possible long-term effects of TTs.

Regrowth and recurrence of symptoms can increase healthcare costs by leading to new contacts with healthcare providers, outpatient visits, and potentially resurgery. In the current study, during the two-year follow-up period the average costs of outpatient visits were greater in the TT group than in the TE group, which might be due to recurrence of symptoms in the TT group. However, resurgery was performed in only $1.5 \%$ of the patients in the TT group, and it is likely that all outpatient contacts will not lead to resurgery. Resurgeries were included in the cost analysis and overall no increase occurred in healthcare costs during the two-year follow-up after TT compared with TE.

The main strength of this study is the relatively large and homogeneous sample, which represents well the Finnish population under the age of 16 years. Another strength is the reliability of the complication data. The families of the children operated on at the Department of ORL are advised to contact our emergency department in case of any adverse event. As our department is the only on-call ORL-HNS center in the HUH area outside office hours, also patients operated on by the private sector or another public hospital in the area contact our emergency department. Therefore, it is unlikely that we would have missed patients with complications. The facts that the data concerning the number of tonsil surgeries and complications were retrieved from two separate databases and that patients were not followed up prospectively 
can be seen as limitations. Because the search was based on ICD diagnosis codes of the emergency department visits, we excluded complications that emerged during the operation and before discharge from hospital. Therefore, immediate complications are not considered. Furthermore, we were not able to limit the cost data specifically to the visits related to tonsil surgery during the two-year follow-up. However, as we included the costs of all otorhinolaryngology specialty visits, we can be confident that the cost analysis did not underestimate the costs.

\section{Conclusion}

The change of the tonsil surgical method from TE to TT in children was associated with a decreased number of tonsil surgery complications, reducing the number of emergency department visits, inpatient days, and postoperative hemorrhages requiring return-to-theatre. In addition, the shift from TE to TT diminished the need for healthcare services and costs. Resurgery after TT was needed by $1.5 \%$ of the patients. Lower morbidity related to tonsil surgery is one contributing factor to tonsil procedures mostly being performed as day-surgeries.

\section{Funding}

The study was supported by the Research Funds of Helsinki University Hospital.

The authors have no financial relationships or conflicts of interest to disclose.

\section{Conflicts of interest}

None.

\section{Acknowledgments}

Carol Ann Pelli is thanked for reviewing the language of the manuscript and Joonas Sakki for assisting with data analysis.

\section{References}

[1] R.A. McNeill, A history of tonsillectomy: two millenia of trauma, haemorrhage and controversy, Ulster Med. J. 29 (1) (1960 Jun) 59-63.

[2] N.P. Parker, D.L. Walner, Trends in the indications for pediatric tonsillectomy or adenotonsillectomy, Int. J. Pediatr. Otorhinolaryngol. 75 (2) (2011 Feb) 282-285.

[3] A. Linder, A. Markstrom, E. Hultcrantz, Using the carbon dioxide laser for tonsillotomy in children, Int. J. Pediatr. Otorhinolaryngol. 50 (1) (1999 Oct 15) 31-36.

[4] E. Hultcrantz, A. Linder, A. Markstrom, Tonsillectomy or tonsillotomy? -A randomized study comparing postoperative pain and long-term effects, Int. J. Pediatr. Otorhinolaryngol. 51 (3) (1999 Dec 15) 171-176.

[5] P.J. Koltai, C.A. Solares, J.A. Koempel, K. Hirose, T.I. Abelson, P.R. Krakovitz, et al.,
Intracapsular tonsillar reduction (partial tonsillectomy): reviving a historical procedure for obstructive sleep disordered breathing in children, Otolaryngol. Head Neck Surg. 129 (5) (2003 Nov) 532-538.

[6] E. Ericsson, J. Graf, E. Hultcrantz, Pediatric tonsillotomy with radiofrequency technique: long-term follow-up, Laryngoscope 116 (10) (2006 Oct) 1851-1857.

[7] E. Hultcrantz, E. Ericsson, Pediatric tonsillotomy with the radiofrequency technique: less morbidity and pain, Laryngoscope 114 (5) (2004 May) 871-877.

[8] J.P. Windfuhr, J.A. Werner, Tonsillotomy: it's time to clarify the facts, Eur. Arch. Oto-Rhino-Laryngol. 270 (12) (2013 Nov) 2985-2996.

[9] E. Odhagen, O. Sunnergren, C. Hemlin, A.C. Hessen Soderman, E. Ericsson, J. Stalfors, Risk of reoperation after tonsillotomy versus tonsillectomy: a population-based cohort study, Eur. Arch. Oto-Rhino-Laryngol. 273 (10) (2016 Oct) 3263-3268.

[10] C.S. Derkay, D.H. Darrow, C. Welch, J.T. Sinacori, Post-tonsillectomy morbidity and quality of life in pediatric patients with obstructive tonsils and adenoid: microdebrider vs electrocautery, Otolaryngol. Head Neck Surg. 134 (1) (2006 Jan) $114-120$.

[11] E. Hultcrantz, A. Linder, A. Markstrom, Long-term effects of intracapsular partial tonsillectomy (tonsillotomy) compared with full tonsillectomy, Int. J. Pediatr. Otorhinolaryngol. 69 (4) (2005 Apr) 463-469.

[12] E. Ericsson, T. Ledin, E. Hultcrantz, Long-term improvement of quality of life as a result of tonsillotomy (with radiofrequency technique) and tonsillectomy in youths, Laryngoscope 117 (7) (2007 Jul) 1272-1279.

[13] R. de la Chaux, C. Klemens, M. Patscheider, O. Reichel, A. Dreher, Tonsillotomy in the treatment of obstructive sleep apnea syndrome in children: polysomnographic results, Int. J. Pediatr. Otorhinolaryngol. 72 (9) (2008 Sep) 1411-1417.

[14] J. Walton, Y. Ebner, M.G. Stewart, M.M. April, Systematic review of randomized controlled trials comparing intracapsular tonsillectomy with total tonsillectomy in a pediatric population, Arch. Otolaryngol. Head Neck Surg. 138 (3) (2012 Mar) 243-249.

[15] J.L. Acevedo, R.K. Shah, S.E. Brietzke, Systematic review of complications of tonsillotomy versus tonsillectomy, Otolaryngol. Head Neck Surg. 146 (6) (2012 Jun) 871-879.

[16] M. Eriksson, U. Nilsson, A.C. Bramhagen, E. Idvall, E. Ericsson, Self-reported postoperative recovery in children after tonsillectomy compared to tonsillotomy, Int. J. Pediatr. Otorhinolaryngol. 96 (2017 May) 47-54.

[17] C. Vicini, M. Eesa, E. Hendawy, M. Pari, G. D'Agostino, S. AlGhamdi, et al., Powered intracapsular tonsillotomy vs. conventional extracapsular tonsillectomy for pediatric OSA: a retrospective study about efficacy, complications and quality of life, Int. J. Pediatr. Otorhinolaryngol. 79 (7) (2015 Jul) 1106-1110.

[18] A. Borgstrom, P. Nerfeldt, D. Friberg, O. Sunnergren, J. Stalfors, Trends and changes in paediatric tonsil surgery in Sweden 1987-2013: a population-based cohort study, BMJ Open 7 (1) (2017 Jan 13) e013346.

[19] R.B. Mitchell, K.D. Pereira, N.R. Friedman, Sleep-disordered breathing in children: survey of current practice, Laryngoscope 116 (6) (2006 Jun) 956-958.

[20] H.H. Patel, C.E. Straight, E.B. Lehman, M. Tanner, M.M. Carr, Indications for tonsillectomy: a 10-year retrospective review, Int. J. Pediatr. Otorhinolaryngol. 78 (12) (2014 Dec) 2151-2155.

[21] E. Foki, R. Seemann, K. Stelter, C. Lill, The effect of tonsillotomy on chronic recurrent tonsillitis in children, Acta Otolaryngol. 137 (9) (2017 Sep) 992-996.

[22] S. Wireklint, E. Ericsson, Health-related quality of life after tonsillotomy versus tonsillectomy in young adults: 6 years postsurgery follow-up, Eur. Arch. Oto-RhinoLaryngol. 269 (8) (2012 Aug) 1951-1958.

[23] J.P. Windfuhr, K. Savva, J.D. Dahm, J.A. Werner, Tonsillotomy: facts and fiction, Eur. Arch. Oto-Rhino-Laryngol. 272 (4) (2015 Apr) 949-969.

[24] O. Sunnergren, E. Odhagen, J. Stalfors, Incidence of second surgery following pediatric adenotonsillar surgery: a population-based cohort study, Eur. Arch. OtoRhino-Laryngol. 274 (7) (2017 Jul) 2945-2951. 